\title{
Complement-activating rheumatoid-factor-containing complexes in patients with rheumatoid vasculitis
}

\author{
C. J. ELSON, D. G. I. SCOTT, D. R. BLAKE, \\ P.A. BACON, AND P. D. J. HOLT
}

From the Departments of Pathology and Medicine, Bristol University; Royal National Hospital for Rheumatic Diseases, Bath; and South Western Regional Transfusion Centre, Bristol

SUMMARY The role of complement and rheumatoid factor in immune complexes was examined in patients with a variety of rheumatic diseases. This was done by assessing the amount of rheumatoid factor (RF) bound from sera by $\mathrm{F}(\mathrm{ab})_{2}$ anti-C3 attached to a solid matrix. High levels of $\mathrm{RF}$ bound to $\mathrm{C} 3$ were detected in patients with rheumatoid arthritis complicated by vasculitis but rarely and in lower levels in patients with synovitis, ankylosing spondylitis, and systemic lupus erythematosus. The activity was bound to anti-C3 through anti-C 3 antibodies because little was bound by normal $\mathrm{F}(\mathrm{ab})_{2}$ and was evidently complexed in the sera before in-vitro testing, since it was precipitated by $2.5 \%$ polyethylene glycol and sedimented with high molecular weight material on sucrose density gradient ultracentrifugation. It is considered that RF-containing complexes are present in vasculitic sera and have the potential to bind complement in vivo.

If rheumatoid factor (RF) is important in the pathogenesis of rheumatoid disease, then increased levels of RF should correlate with at least some aspect of disease activity. We have shown that high levels of serum IgG RF are strongly associated with the incidence of vasculitis but not with synovitis. ${ }^{1}$ Studies of the temporal relationship of serum IgG RF levels to anticomplementary activity, C4 levels, and clinical features of vasculitis revealed that IgG RF levels and anticomplementary activity rose while $C 4$ levels fell with clinical relapse and that the levels returned to normal with clinical improvement. ${ }^{2}$ Our interpretation of these results is that antigen-antibody complexes containing IgG (and probably in addition $\mathrm{IgM}^{3}$ ) RF activate complement, thereby initiating inflammation. It follows that RF-containing complexes capable of activating complement should be found in sera from rheumatoid patients with vasculitis but not synovitis. This was investigated by incubating sera from these patients with the $\mathrm{F}(\mathrm{ab})_{2}$ fragments of anti-human $\mathrm{C} 3$ attached to a solid matrix. C3 together with any material which has bound C 3 should be taken up by the matrix. The presence of any bound RF was assayed by adding ${ }^{125}$ I-labelled heat-aggregated IgG.

\section{Materials and methods}

PATIENTS

Blood was collected from patients attending rheumatology outpatient clinics or admitted to

Accepted for publication 15 February 1982.

Correspondence to Dr C. J. Elson, Immunology Group, Department of Pathology, University of Bristol, Bristol BS8 1TD. hospital for severe rheumatic disease. Sera were separated and stored at $-20^{\circ} \mathrm{C}$ until tested. Patients with rheumatoid arthritis (RA) fulfilled the American Rheumatism Association criteria for classical or definite RA. Vasculitis was diagnosed by clinical findings of nail fold, nail edge, or digital infarcts, deep cutaneous ulceration, or neuropathy. Histological evidence of vasculitis was confirmed in most cases by either skin or rectal biopsy and was necessary for inclusion in the absence of typical clinical features. Current drug therapy was noted, and the results are from patients whose samples were taken before treatment for vasculitis. Other rheumatic diseases defined by accepted clinical and radiological criteria were ankylosing spondylitis and systemic lupus erythematosis (SLE). Fifteen of the 20 patients with ankylosing spondylitis had active peripheral joint synovitis. Normal control sera were from healthy volunteers.

\section{ESTIMATION OF RF ACTIVITY BOUND BY AN T I-C 3}

$\mathrm{F}(\mathrm{ab})_{2}$ fragments of antihuman C3 $(100 \mu \mathrm{g} / \mathrm{ml})(100$ $\mathrm{mg} / \mathrm{l})$ were incubated in the wells of polyvinyl microtitre plates (Linbro) for an hour at $37^{\circ} \mathrm{C}$ and overnight at $4^{\circ} \mathrm{C}$. Unbound anti-C3 was removed, the plates washed, and $1 \%$ bovine serum albumin (BSA) added to the wells to block any free reactive sites. After incubating for 2 hours at $37^{\circ} \mathrm{C}$ the plates were washed and stored (up to 2 months) at $4^{\circ} \mathrm{C}$ until required. Dilutions of sera $1: 125$ were added to the wells, the plates incubated for 2 hours at $37^{\circ} \mathrm{C}$, and washed. Heat aggregated $\left(63^{\circ} \mathrm{C}\right.$ for 30 minutes ${ }^{125} \mathrm{I}-$ 
labelled rabbit (or human $\mathrm{IgG}$ ) at $1 \mu \mathrm{g} / \mathrm{ml}(1 \mathrm{mg} / \mathrm{l})$ was added to the wells, the plates incubated for 2 hours at room temperature, and then washed. Counts were made of the radioactivity bound to each well. Counts obtained from control wells incubated with $1 \%$ BSA alone followed by labelled IgG were subtracted from each sample count. The proportion of the total labelled IgG bound by test samples was calculated and the results expressed as $\mu \mathrm{g}$ labelled $\mathrm{IgG}$ bound per $\mathrm{ml}$ of serum.

\section{REAGENTS}

IgG was prepared from normal human or rabbit serum (or from rabbit antisera) by precipitation with $20 \%$ sodium sulphate followed by elution from a diethylaminoethyl sepharose column and gel filtration on AcA 44 (LKB).

$\mathrm{F}(\mathrm{ab})_{2}$ fragments were prepared by digestion with pepsin followed by gel filtration on AcA 44. The efficacy of pepsin digestion was tested by radioassay with ${ }^{125}$ I-labelled goat antirabbit Fcy antibody. ${ }^{3}$ Small amounts of this antibody bound to wells coated with $\mathrm{F}(\mathrm{ab})_{2}$ preparations. By contrast high levels bound to wells coated with intact IgG.

Antisera specific to human $\gamma$ and $\mu$ were prepared as described previously. ${ }^{3}$ Antihuman $\mathrm{C} 3$ was raised in rabbits by injecting them with their own erythrocytes which had been incubated in vitro with human serum, as described by Merry and colleagues. ${ }^{4}$ It was tested by 2-dimensional electrophoresis against ethylene diamine tetra-acetate treated human plasma and 2-day-old serum and by direct passive haemagglutination tests against human erythrocytes coated with various human $\mathrm{C} 4$ and $\mathrm{C} 3$ subcomponents. $^{5-7}$ The specificity of the antiserum was for C3c and C3d. It was passed down human IgG sepharose and the $F(a b)_{2}$ fragments prepared before use.

Separation of sera by sucrose density gradient ultracentrifugation and the assay for IgG rheumatoid factor have been described. ${ }^{3}$

\section{Results}

Sera from normal subjects and 4 groups of rheumatic patients were examined for their capacity to bind labelled heat-aggregated rabbit IgG after incubation on anti-C3 coated plates (Fig. 1). Binding was detected in all $(16 / 16)$ sera from patients with rheumatoid vasculitis but only in $6 / 28$ sera from patients with synovitis, and these sera had relatively low activity. Sera from 9 normal subjects, 12 patients with ankylosing spondylitis, and 12 with SLE had little or no activity. Tests carried out with labelled heat-aggregated human IgG gave the same results. Some sera were frozen and thawed on a number of occasions before testing, but this did not alter their

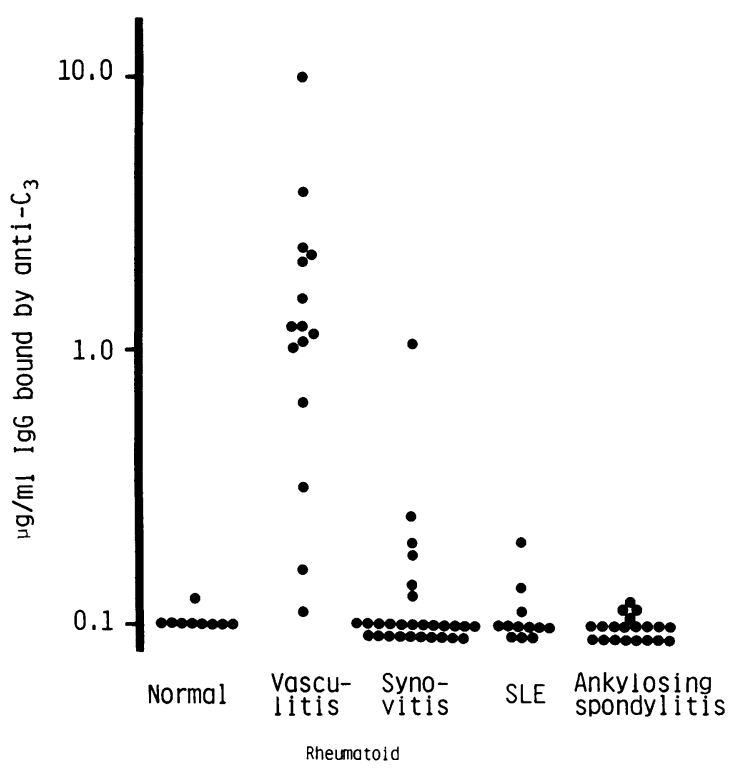

Fig. 1 Estimate of RF activity bound by anti-C3 from sera ofnormal subjects and patients with $R A$ and other arthritides.

ability to take up labelled rabbit IgG on anti-C3 coated plates. It was important to test that the RF activity attached to the solid phase was bound by anti-C 3 antibodies. This was done by comparing the uptake of labelled IgG on rabbit $\mathrm{F}(\mathrm{ab})_{2}$ anti-C3 plates, incubated with sera, with the uptake on normal rabbit $\mathrm{F}(\mathrm{ab})_{2}$ coated plates incubated with the same sera (Table 1). Only one serum (from patient 1 ) bound more than $20 \%$ labelled IgG on normal $\mathrm{F}(\mathrm{ab})_{2}$ coated plates as compared with anti-C3 coated plates. So far we have tested over 250 sera from more than 50 patients, and serum from this patient is exceptional.

Experiments were set up to determine if the rheumatoid factor activity bound by anti-C 3 from

Table.1 Comparison of IgG binding activity by sera on $F(a b)_{2}$ anti-C3 plates with activity on normal $F(a b)_{2}$ plates

\begin{tabular}{|c|c|c|c|}
\hline \multirow[t]{2}{*}{ Patient } & \multicolumn{2}{|c|}{$\mu g / m l ~ I g G$ bound by sera on } & \multirow{2}{*}{$\begin{array}{l}\% F(a b)_{2} / \\
\text { anti-C3 }\end{array}$} \\
\hline & Anti-C3 & $F(a b)_{2}$ & \\
\hline 1 & 10 & 4 & 40 \\
\hline 2 & $1 \cdot 3$ & $0 \cdot 1$ & 8 \\
\hline 3 & $5 \cdot 9$ & $1 \cdot 2$ & 20 \\
\hline 4 & 0.7 & $0 \cdot 1$ & 14 \\
\hline 5 & $1 \cdot 4$ & $0 \cdot 1$ & 7 \\
\hline 6 & $3 \cdot 6$ & 0.6 & 16 \\
\hline 7 & $1 \cdot 1$ & $0 \cdot 1$ & 9 \\
\hline 8 & $1 \cdot 8$ & $0 \cdot 2$ & 11 \\
\hline 9 & $2 \cdot 7$ & $0 \cdot 2$ & 7 \\
\hline
\end{tabular}

SI conversion: $\mu \mathrm{g} / \mathrm{ml}=\mathrm{mg} / \mathrm{l}$. 
Table 2 Effect of precipitation with $2.5 \%$ polyethylene glycol $(P E G) *$ on RF activity bound by anti-C3 from vasculitic sera and IgG RF activity

\begin{tabular}{|c|c|c|c|c|}
\hline & \multicolumn{2}{|c|}{$\begin{array}{l}\mu g / m l \lg G \text { bound by sera } \\
\text { on anti-C3 }\end{array}$} & \multicolumn{2}{|c|}{$I g G R F \mathrm{mg} / \mathrm{ml}$} \\
\hline & Serum & PEG precipitate & Serum & PEG precipitate \\
\hline 1 & 1.9 & $2 \cdot 4$ & 3.0 & $3 \cdot 5$ \\
\hline 2 & $2 \cdot 5$ & $1 \cdot 6$ & $3 \cdot 5$ & $3 \cdot 9$ \\
\hline 3 & 1.6 & 1.9 & $2 \cdot 4$ & Not tested \\
\hline 4 & $4 \cdot 5$ & $2 \cdot 0$ & $4 \cdot 0$ & $4 \cdot 6$ \\
\hline
\end{tabular}

*The PEG precipitates from sera were washed once in $2.5 \%$ PEG, redissolved, and made up to their original volume in phosphate buffered saline. SI conversion: $\mu \mathrm{g} / \mathrm{ml}=\mathrm{mg} / \mathrm{l}$.

Table 3 Influence of $F(a b)_{2}$ anti- $\gamma, F(a b)_{2}$ anti- $\mu$ and normal $F(a b)_{2}$ on uptake of rabbit IgG by sera on anti-C3 coated plates

\begin{tabular}{lllll}
\hline Serum & \multicolumn{4}{l}{$\mu g / m l I_{g} G$ bound by sera on anti-C3 in the presence of: } \\
\cline { 2 - 5 } & - & $F(a b)_{2}$ anti- $\gamma$ & $F(a b)_{2}$ anti- $\mu$ & normal $F(a b)_{2}$ \\
\hline 1 & $8 \cdot 0$ & $6 \cdot 2$ & $8 \cdot 5$ & $7 \cdot 1$ \\
2 & $3 \cdot 1$ & $2 \cdot 2$ & $3 \cdot 4$ & $2 \cdot 7$ \\
3 & $0 \cdot 3$ & $0 \cdot 1$ & 0.4 & $0 \cdot 2$ \\
\hline
\end{tabular}

SI conversion: $\mu \mathrm{g} / \mathrm{ml}=\mathrm{mg} / \mathrm{l}$.

vasculitic sera was complexed in the sera before invitro testing. Table 2 shows that both RF activity bound by anti-C 3 and IgG RF activity were precipitated by $2.5 \%$ polyethylene glycol. Some sera were also ultracentrifuged on sucrose density gradients and the fractions tested for RF activity bound by anti-C3. The activity was restricted to the high (greater than $19 \mathrm{~S}$ ) molecular weight containing fractions.

Tests were carried out to determine the isotype of the rheumatoid factor bound by anti-C 3 from vasculitic sera. Sera were incubated with anti-C 3 coated plates, the plates washed, and labelled rabbit IgG added to the plates alone or in the presence of rabbit $\mathrm{F}(\mathrm{ab})_{2}$ antihuman $\gamma$, rabbit $\mathrm{F}(\mathrm{ab})_{2}$ antihuman $\mu$ or normal rabbit $\mathrm{F}(\mathrm{ab})_{2}$. At low concentrations no effect was observed, but at $50 \mu \mathrm{g} / \mathrm{ml}(50 \mathrm{mg} / \mathrm{l})$ (Table 3) IgG uptake was partially inhibited by anti- $\gamma$, although part of this effect appears to be non anti- $\gamma$ specific, because there was some inhibition by normal $\mathrm{F}(\mathrm{ab})_{2}$. Curiously, anti- $\mu$ if anything increased IgG uptake.

\section{Discussion}

Previous results showed that high levels of IgG RF and anticomplementary activity are coincident in the sera of patients with rheumatoid vasculitis ${ }^{2}$ and also that complexes containing both IgG RF and IgM RF are present in these sera. ${ }^{3}$ However, it could only be inferred that the complement-fixing complexes were those which contained RF. The method described here was devised to test this. We reasoned that complexes containing RF and complement (but not free RF or other RF containing complexes) would be bound by anti-C 3 and bind IgG through any RF with unoccupied antigen-binding sites. On the other hand non-RF-containing complexes bound by anti-C3 would not take up IgG. Thus the results which show that $\mathrm{IgG}$ reacted strongly with anti-C3 coated plates incubated with sera from patients with vasculitis but poorly with sera from patients with synovitis support the hypothesis. This conclusion depends on 2 assumptions which were tested. The first is that the RF-containing complexes were bound to anti-C3 through its anti-C3 activity. The fact that little RF activity was bound by normal rabbit $F(a b)_{2}$ coated plates supports this. The second is that the RF was complexed before addition to the plates rather than by non-RF-containing complexes being bound by anti-C3 and RF then binding to any IgG in the complex. The former seems likely, because $2.5 \%$ polyethylene glycol, which precipitates neither monomeric IgG nor $\operatorname{IgM}^{8}$ and should therefore separate free from unbound RF, did precipitate the RF activity bound by anti-C 3 . This finding, together with the fact that the RF activity bound by anti-C3 sedimented with material of molecular weight of over 900000 , also suggests that C3 was bound to the RF-containing complex before in-vitro testing, because free C3 (MW 200 000) should be separated from the complex by these procedures.

Attempts to determine the isotype(s) of the RF which was bound by anti-C 3 from vasculitis sera were less successful. There was only some inhibition of uptake of labelled IgG by $\mathrm{F}(\mathrm{ab})_{2}$ anti- $\gamma$ and none by anti- $\mu$. It was expected that inhibition by a particular heavy-chain specific anti-serum would indicate the presence of RF of the corresponding isotype. The finding that RF activity bound by anti-C 3 sedimented with high molecular weight material could accord with the presence of complexed IgG RF, as this is where the bulk of IgG RF sediments ${ }^{3}$ but could also be due to complexed IgM RF.

It may be asked if complement reacted with RF while the sera were collected and stored. Against this many of the sera were thawed only once before testing, and repeated freezing and thawing did not alter the results. Alternatively, complement may have reacted in vivo. Even if this is not so, it is clear that in vasculitic sera RF-containing complexes have the potential to bind complement in vivo and thereby be the vehicle for their own deposition in blood vessel walls, perhaps by releasing pharmacological mediators of vascular permeability. ${ }^{9}{ }^{10}$ Once deposited in blood vessel walls, these complementactivating complexes may be further involved in the 
development of inflammation ${ }^{11}$ by localising it to the site of their deposition.

This work was supported by grants from the Nuffield Foundation and Medical Research Council.

\section{References}

1 Allen C, Elson C J, Scott D G I, Bacon P A, Bucknall R C. IgG anti-globulins in rheumatoid arthritis and other arthritides: relationship to clinical features and other parameters. Ann Rheum Dis 1981; 40: 127-31.

2 Scott D G I, Bacon P A, Allen C, Elson C J, Wallington T. IgG rheumatoid factor, complement and immune complexes in rheumatoid synovitis and vasculitis: comparative and serial studies during cytotoxic therapy. Clin Exp Immunol 1981; 43: 54-63.

3 Jones V E, Cowley P J, Allen C, Elson C J. The isolation of immune complexes IgM rheumatoid factor and recovery of IgG rheumatoid factors from the complexes. J Immunol Methods 1980; 37: 1-14.
4 Merry A H, Rawlinson V I, Stratton F. A simple method for the production of anti-C3 antisera in rabbits. Vox Sang 1980; 38: 288-35.

5 Stratton F, Rawlinson V I. Preparation of test cells for the anti-globulin test. J Clin Pathol 1974; 27: 359-67.

6 Fruitstone M J. C3b-sensitised erythrocytes (cited in correspondence to editor). Transfusion 1978; 18: 125.

7 Freedman J. Mollison P L. Preparation of red cells coated with $\mathrm{C} 4$ and $\mathrm{C} 3$ subcomponents and production of anti-C4d and anti-C3d. Vox Sang 1976; 31: 241-57.

8 Creighton W D, Lambert P H, Miesher P A. Detection of antibodies and soluble antigen-antibody complexes by precipitation with polyethylene glycol. J Immunol 1973; 111: 1219-27.

9 Cochrane C J, Hawkins D. Studies on circulating immune complexes III. Factors governing the ability of circulating complexes to localize in blood vessels. J Exp Med 1968; 127: 137.

10 Wedmore C V, Williams T J. Control of vascular permeability by polymorphonuclear leukocytes in inflammation. Nature 1981; 289: 646-50.

11 Scott D G I, Bacon P A, Tribe C R. Systemic rheumatoid vasculitis: a clinical and laboratory study of 50 cases. Medicine (Baltimore) 1981; 60: 288-97. 